\title{
DERECHOS DE LOS PUEBLOS INDÍGENAS, PLURALISMO JURÍDICO Y DEFENSA CULTURAL EN EL ÁMBITO PENAL. REFLEXIONES A PARTIR DEL CASO MEXICANO*
}

\author{
RIGHTS OF INDIGENOUS PEOPLES, LEGAL PLURALISM AND \\ CULTURAL DEFENSE IN THE CRIMINAL FIELD. REFLECTIONS \\ STARTING FROM THE MEXICAN CASE
}

\author{
Paola Parolari \\ Università degli Studi di Brescia (Bréscia, Itália) \\ Recebimento: 22 maio 2019 \\ Aceitação: 14 ago. 2019
}

\begin{abstract}
Como citar este artigo / How to cite this article (informe a data atual de acesso / inform the current date of access):
PAROLARI, Paola. Derechos de los pueblos indígenas, pluralismo jurídico y defensa cultural en el ámbito penal. Reflexiones a partir del caso mexicano. Revista da Faculdade de Direito UFPR, Curitiba, PR, Brasil, v. 64, n. 3, p. 930, set./dez. 2019. ISSN 2236-7284. Disponível em: https://revistas.ufpr.br/direito/article/view/67009. Acesso em: 11 mar. 2020. DOI: http://dx.doi.org/10.5380/rfdufpr.v64i3.67009.
\end{abstract}

\section{RESUMEN}

Varias constituciones nacionales y declaraciones o convenciones internacionales reconocen el derecho a la diversidad cultural como un derecho fundamental. Al mismo tiempo, en las sociedades multiculturales existe la posibilidad de que individuos con culturas diferentes consideren, como lícitas, conductas sancionadas penalmente por el derecho estatal. Se discute, entonces, si los factores culturales que han condicionado la conducta del imputado deberían tener relevancia en la valoración de su responsabilidad penal, tanto en los contextos de inmigración como en aquellos Estados en los que hay pueblos indígenas. Centrándose en el segundo caso, el artículo quiere verificar la viabilidad y deseabilidad de dos posibles medios de reconocimiento de las diferencias culturales en el ámbito penal: el pluralismo jurídico y la defensa cultural. Para este propósito, se toma en consideración el ejemplo de cómo se configuran estos medios en el ordenamiento jurídico mexicano. Luego se discute, en una perspectiva más general y teórica, los “desafíos técnicos” que plantean - bajo el perfil jurídico, institucional y socio-antropológico - tanto la constitución de un régimen de pluralismo jurídico, como el recurso a la defensa cultural en los tribunales estatales. Finalmente, los argumentos normativos más recurrentes a favor y en contra del pluralismo cultural en el ámbito penal son sometidos a un examen crítico. El análisis lleva a la conclusión de que, aunque la identificación de posibles “equilibrios avanzados” entre respeto por las diferencias culturales y tutela de los derechos fundamentales en el ámbito penal es un objetivo difícil de alcanzar, no es, sin embargo, a priori imposible.

\footnotetext{
* El contenido de este artículo se presentó oralmente durante un homónimo seminario interno de estudio celebrado el 19 de septiembre de 2018 en la Escuela Judicial Electoral del Tribunal Electoral del Poder Judicial de la Federación, Ciudad de México. Agradezco al Dr. Carlos A. Soriano Cienfuegos y al Dr. José Ramón Narváez Hernández por su invitación.
} 


\title{
PALABRAS CLAVE
}

Diversidad cultural. Derecho penal. Derechos de los pueblos indígenas. Pluralismo jurídico. Defensa cultural.

\begin{abstract}
Several national constitutions and international legal instruments recognize the right to cultural diversity as a fundamental right. At the same time, in multicultural societies there is the possibility that individuals from different cultures consider, as licit, behaviors that are criminally sanctioned by the law of the state. It is therefore discussed whether the cultural factors which conditioned the behavior of the defendant should be taken into account in the assessment of her/his criminal responsibility, both in immigration contexts and in those states where there are indigenous peoples. Focusing on the second case, the article aims to verify the feasibility and the desirability of two possible means of recognition of cultural differences in the criminal field: legal pluralism and the cultural defense. To this purpose, it considers the example of how these means are configured in the Mexican legal order. It then discusses, in a more general and theoretical perspective, the "technical" challenges posed - at the legal, institutional and even socio-anthropological level - both by the constitution of a regime of legal pluralism and by the use of the cultural defense in state courts. Finally, the most recurrent normative arguments for and against cultural pluralism in the criminal field are subjected to critical examination. The analysis leads to the conclusion that, although the identification of possible "advanced balances" between respect for cultural differences and the protection of fundamental rights in the criminal field is a difficult goal to achieve, it is not, however, impossible a priori.
\end{abstract}

\section{KEYWORDS}

Cultural Diversity. Criminal law. Rights of indigenous peoples. Legal pluralism. Cultural defense.

\section{INTRODUCCIÓN}

Desde el periodo de la segunda posguerra, se multiplican las declaraciones y las convenciones internacionales que reconocen explícitamente el derecho a la diversidad cultural como un derecho fundamental. Piénsese, por ejemplo, en la Declaración sobre la raza y los prejuicios raciales (UNESCO, 1978), el Convenio sobre pueblos indígenas y tribales en países independientes (Convenio 169 de la OIT, 1989), la Declaración de principios sobre la tolerancia (UNESCO, 1995), la Declaración universal sobre la diversidad cultural (UNESCO, 2001), la Convención sobre la protección y promoción de la diversidad de las expresiones culturales (UNESCO, 2005) y la Declaración sobre los derechos de los pueblos indígenas (ONU, 2007)². Además, en los últimos

\footnotetext{
${ }^{1}$ Como afirma MAZZARESE (2014; 2018), todas estas declaraciones y convenciones son expresiones particulares de un más general derecho a ser diversos como principio fundamental y fundador del constitucionalismo (inter)nacional que se estableció después de la Segunda Guerra Mundial. En este sentido, sobre el falso contraste entre igualdad en los derechos fundamentales y tutela de las diferencias (culturales), véanse FERRAJOLI (2007, vol. 2, págs. 57-61; 2018, págs. 36-69); GIANFORMAGGIO (2005, págs. 33-61, 85-105).
} 
años, también un número creciente de Estados ha reconocido el derecho a la diversidad cultural de individuos y grupos en sus cartas constitucionales ${ }^{2}$.

Simétricamente, en las sociedades multiculturales, cada vez más individuos y grupos exigen al Estado el reconocimiento y la tutela jurídica de sus especificidades culturales. Por lo tanto, la tutela de los derechos fundamentales está llamada a medirse con la existencia de una pluralidad de tradiciones y valores a menudo muy diferentes de aquellos que informan el derecho estatal. Sin embargo, en el ámbito penal, la dificultad de conciliar diferentes concepciones de la vida y del bien asume, a menudo, acentos de especial gravedad, porque al derecho penal se le ha reconocido la función de sancionar específicamente (y exclusivamente) las violaciones a los bienes jurídicos considerados como fundamentales e irrenunciables; bienes, por ejemplo, como la vida, la integridad física o la libertad individual.

Una cuestión que se discute mucho, en particular, es la de la posible relevancia penal de la identidad cultural del imputado en situaciones de conflicto normativo en las cuales, en razón de la propia diversidad cultural, el imputado se siente vinculado a normas morales, sociales, o incluso jurídicas, que chocan con el derecho penal estatal. De hecho, el carácter multicultural de las sociedades nacionales implica la posibilidad de que individuos con culturas diferentes consideren moralmente impuestas, socialmente toleradas, o incluso jurídicamente lícitas, conductas que son en cambio penalmente sancionadas por el Estado. Se desvela, así, la ficción de la homogeneidad de valores morales, sociales y jurídicos dentro de las sociedades nacionales; homogeneidad que, sin embargo, es generalmente asumida por el sistema penal estatal. Se cuestiona, entonces, si la identidad cultural del imputado debe influir sobre la valoración de su responsabilidad penal.

En los Estados Unidos se discute al respecto por lo menos desde la mitad de los años ochenta del siglo XX, como consecuencia de sentencias que, más o menos informalmente, han reconocido un valor atenuante a la influencia de factores culturales en la determinación de la conducta penalmente relevante del imputado (Véanse, por ejemplo, LYMAN, 1986; SHEYBANI, 1987; OLIVER, 1988; YOUNG, 1992; VOLPP, 1994; COLEMAN, 1996; FISCHER, 1998; SIKORA, 2001; GOLDING, 2002; RENTELN, 2004). Pero esta cuestión no se impone, obviamente, solo en los Estados Unidos, sino también en Europa y en otros países en los que existe inmigración: no en vano, la literatura sobre el tema se ha difundido en varios países europeos (Véase, por ejemplo: en Italia, MONTICELLI,

\footnotetext{
${ }^{2}$ En América Latina, piénsese en las reformas relacionadas con los pueblos indígenas introducidas en las cartas constitucionales de muchos países desde la mitad de los años ochenta, en el marco de lo que ha sido denominado "nuevo constitucionalismo latinoamericano”. Véase, entre otros, UPRIMNY (2011, págs. 1589-1594, 1603-1604). Mas en general, sobre la relevancia de la diversidad cultural en diversas constituciones nacionales en el mundo, véase RUGGIU (2012, págs. 217-235).
} 
2003; BERNARDI, 2006 y 2007; BASILE, 2008; DE MAGLIE, 2010; PAROLARI, 2011; en Francia, FOBLETS, 1998; en Inglaterra, PHILLIPS, 2003; en España, PÉREZ DE LA FUENTE, 2012; SANZ MULAS, 2018. Más en general, ensayos sobre la defensa cultural en diversos países están colectados en FOBLET y RENTELN, Coords., 2009).

Además, esta cuestión se impone en los países en los que hay pueblos indígenas (En América Latina, véanse, por ejemplo, entre muchos otros, SOTOMAYOR ACOSTA, 1996; HURTADO POZO, 2001; VALENZUELA REYES, 2003; CARNEVALI, 2007; CASTILLO ARA, 2014; LILLO, 2015; LÓPEZ BETANCOURT y FONSECA LUJÁN, 2016. Además, sobre la relación entre política criminal estatal e indigenismo véase MAUREIRA PACHECO, 2005). En México, por ejemplo, el debate sobre la relevancia de la identidad cultural del imputado se ha centrado precisamente en la cuestión de cómo conciliar la pretensa punitiva del Estado en el ámbito penal, con el derecho de los pueblos indígenas a su propia especificidad cultural, como reconocido no solo en la Constitución política de los estados unidos mexicanos sino también, específicamente, en el Código nacional de procedimientos penales.

De todas formas, lo que pasa es que, en una pluralidad de Estados diferentes, se han registrado decisiones judiciales en las que la cultura del imputado ha sido objeto de valoración y ha sido tomada en cuenta, sea para atenuar, sea para agravar, su responsabilidad penal ${ }^{3}$. Esta tendencia cada vez más difundida de atribuir relevancia jurídica a la influencia de factores culturales en la formación de la motivación para delinquir se inserta en un proceso, aún contradictorio en sus expresiones e incierto en sus éxitos, a través del cual los ordenamientos jurídicos estatales reaccionan, e intentan dar respuesta, a los conflictos que contraponen sistemas normativos diferentes al interior del territorio del Estado.

En este trabajo, mi propósito es: identificar las formas que el reconocimiento de las identidades culturales podría asumir en el ámbito penal, con especial referencia a los estados en los que hay pueblos indígenas ( $(1)$; mencionar en cual dirección se va moviendo el ordenamiento jurídico mexicano (§ 2); analizar los desafíos “técnicos” que pueden complicar el reconocimiento de las identidades culturales en el ámbito penal (§ 3); y, finalmente, debatir las razones en favor y en contra de este reconocimiento (§ 4).

\footnotetext{
${ }^{3}$ En este trabajo solo se considera la posibilidad de un efecto atenuante. Sobre los problemas conectados a los efectos agravantes véanse BERNARDI (2007) y PAROLARI (2011).
} 


\section{LOS DELITOS CULTURALMENTE MOTIVADOS: ¿DEFENSA CULTURAL O PLURALISMO JURÍDICO E INSTITUCIONAL?}

Para dar cuenta de los condicionamientos culturales que pueden caracterizar delitos cometidos por personas pertenecientes a culturas diversas de aquella que informa el sistema penal del Estado, la doctrina acude a la categoría de los “delitos culturalmente motivados” (VAN BROECK, 2001). Entre estos delitos no se comprende cualquier delito cometido por inmigrantes, miembros de pueblos indígenas o personas que de algún modo pertenecen a grupos cuya cultura se diferencia de aquella que informa el sistema penal del Estado. Pueden, de hecho, calificarse como culturalmente motivados solamente aquellos tipos penales concretos en los que el imputado haya violado la ley penal precisamente por el hecho de haber actuado conforme a los preceptos morales, a las tradiciones sociales o a las normas jurídicas del grupo cultural al que pertenece. Para que un delito pueda decirse culturalmente motivado es necesario, entonces, que la conducta delictuosa sea en efecto jurídicamente y/o socialmente y/o moralmente lícita, o al menos tolerada, en la cultura a la que pertenece el imputado, y que este último haya, en efecto, cometido el delito en razón de los condicionamientos de su propia cultura.

Los condicionamientos culturales pueden afectar la conducta del imputado de diferentes maneras (RENTELN, 2010, págs. 87-89). Tal vez, el imputado no se da cuenta de que su comportamiento está sancionado por el derecho penal, debido a un error directo o indirecto de prohibición. Es decir, el imputado ignora la antijuridicidad de sus acciones o está convencido que su actuar se dispensa por una causa de justificación reconocida por el derecho penal estatal. Hay veces, en cambio, en las que el imputado sabe que sus acciones son ilícitas, pero igualmente las realiza, porque se siente obligado a respetar las normas jurídicas, sociales o morales de su grupo cultural. Finalmente, hay casos en los que el imputado sabe que sus acciones son ilícitas, pero igualmente las realiza, porque ha recibido provocaciones que, de acuerdo con su cultura, son tan graves como para justificar su reacción.

Frente a los delitos culturalmente motivados, la idea del reconocimiento de las diversidades culturales en el ámbito penal plantea al menos dos cuestiones diferentes. La primera es la cuestión de la elección del derecho aplicable. La segunda es la cuestión de los sujetos institucionales que tienen la competencia para aplicar y garantizar el respeto de este derecho.

La primera cuestión podría parecer absurda frente a la idea dominante de que el derecho penal estatal es el único derecho penal legítimo. Pero hay que tomar en cuenta que también los sistemas normativos de los grupos culturales pueden incluir normas, en sentido amplio, penales. Este tema es particularmente relevante, por ejemplo, en el caso de los pueblos indígenas. Por lo tanto, es 
necesario, antes que nada, establecer si el derecho a la diversidad cultural incluye el derecho a seguir sus propios sistemas normativos, incluso los jurídicos; es decir, si hay un derecho al pluralismo jurídico ${ }^{4}$, entendido como una estructura institucional caracterizada por relaciones de reconocimiento (mutuo) entre sistemas jurídicos distintos. Mas en particular, hay que establecer en qué ámbitos se deben aplicar, eventualmente, las normas de los pueblos indígenas, y si estos ámbitos pueden o deben incluir el derecho penal.

Si se admite que hay un derecho de las personas pertenecientes a pueblos indígenas a seguir sus propios sistemas jurídicos, la segunda cuestión es decidir qué instituciones deben garantizar el respeto y la aplicación de las normas aplicables. En particular, es dudoso si es preferible idear e implementar una división entre los ámbitos en los cuales el Estado ejerce su jurisdicción y aquellos en los que interviene la jurisdicción de los pueblos indígenas, o si la resolución de las disputas debe reservarse en cualquier caso a los tribunales estatales, independientemente del derecho aplicable. En el primer caso, se trata de crear formas de gobernanza compartida entre el Estado y los pueblos indígenas, según lo que Ayelet Shachar ha definido "jurisdicciones multiculturales” (SHACHAR, 2001). En el segundo caso, la forma de reconocer las especificidades culturales de los indígenas en los tribunales estatales puede ser la de introducir en el derecho penal estatal normas que permitan, o incluso obliguen, al juez a tomar en cuenta la identidad cultural del imputado y los consiguientes conflictos normativos en los que él se puede encontrar. Dicho de otra forma, este tipo de reconocimiento coincide con la introducción de la que ha sido llamada “defensa cultural”.

La expresión “defensa cultural” (cultural defense) ha sido introducida por la doctrina estadounidense precisamente para indicar una particular categoría de argumentos defensivos utilizados para pedir y/o conceder una atenuación, o incluso la exclusión, de la pena en consideración de la identidad cultural del imputado ${ }^{5}$. Genéricamente, la defensa cultural remite a la idea de que personas de cultura diversa no pueden ser consideradas plenamente responsables por una conducta sancionada por el derecho penal estatal, si es que han actuado de conformidad con normas morales, sociales o jurídicas del propio grupo cultural. En sentido técnico, la defensa cultural es una categoría doctrinal pensada para denominar una institución iuspenalistica que, en presencia de determinadas

\footnotetext{
${ }^{4}$ Sobre la ambigüedad del derecho internacional de los derechos humanos en relación con el pluralismo jurídico, véase BREMS (2017). Sobre la idea de un derecho al pluralismo jurídico véase, entre otros, CAMMARATA (2010; 2012, pág. 146).

${ }^{5}$ Numerosos son los casos vueltos celebres por la doctrina estadounidense y retomados también por comentadores y estudiosos europeos. Entre los principales: People v. Kimura, People v. Chen, People v. Moua. Para una gran serie de casos, véase, en particular, RENTELN (2004). Cabe recordar que la noción de defensa cultural ha sido elaborada, en origen, con referencia a los inmigrantes. Sin embargo, ha sido utilizada, en seguida, también con referencia a los indígenas.
} 
condiciones, identificase en el background cultural del imputado una causa de justificación o una circunstancia atenuante de su responsabilidad penal. En todo caso, la condición para la aplicación de la defensa cultural es el carácter culturalmente motivado del delito cometido.

Dependiendo del caso concreto, puede ser que la defensa cultural sea utilizada para excluir la imputabilidad del imputado (por ejemplo, por error de comprensión de los hechos), para excluir su culpabilidad (por ejemplo, en el caso de error directo o indirecto de prohibición ${ }^{6}$ ) o como circunstancia modificativa de la responsabilidad (atenuante de la pena, por ejemplo, en el caso de la provocación) ${ }^{7}$. Cabe considerar, sin embargo, una posibilidad más: es decir, que la defensa cultural se traduzca en una causa de justificación que excluye la misma antijuridicidad del hecho, cada vez que el imputado haya actuado conforme a las normas jurídicas de su grupo cultural (LÓPEZ BETANCOURT y FONSECA LUJÁN, 2016, págs. 34 ss. Véase § 2). En este sentido, la defensa cultural se volvería en una expresión del derecho al pluralismo jurídico en el ámbito penal.

\section{EL DERECHO A LA IDENTIDAD CULTURAL DE LOS PUEBLOS INDÍGENAS EN EL DERECHO (PENAL) MEXICANO}

El ordenamiento jurídico federal mexicano es un caso interesante para discutir el tema del reconocimiento de las identidades culturales en el ámbito penal, ya que, como se ha dicho, reconoce explícitamente el derecho a la diversidad cultural de los pueblos indígenas no solo en la Constitución política de los estados unidos mexicanos, sino también específicamente en unas normas del Código nacional de procedimientos penales ${ }^{8}$.

En particular, el apartado A, del artículo 2 constitucional "reconoce y garantiza el derecho de los pueblos y las comunidades indígenas a la libre determinación y, en consecuencia, a la autonomía para”, entre otras cosas,

I. Decidir sus formas internas de convivencia y organización social, económica, política y cultural.

II. Aplicar sus propios sistemas normativos en la regulación y solución de sus conflictos internos, sujetándose a los principios generales de esta Constitución, respetando las garantías

\footnotetext{
${ }^{6}$ Sobre el concepto de "error de comprensión culturalmente condicionado" como subcategoría del error de prohibición en el ámbito penal, con referencia a los miembros de pueblos indígenas en México, véase LÓPEZ BETANCOURT y FONSECA LUJÁN (2016, págs. 32-33). Más en general, sobre la ponderación de las valoraciones culturales en el error de prohibición, CASTILLO ARA (2014) proporciona dos distinciones fundamentales: a) entre conocimiento, comprensión e internalización de la prohibición penal; y b) entre error de prohibición vencible e invencible.

${ }^{7}$ Sobre diferentes formas de articular la defensa cultural véanse, por ejemplo, CARNEVALI (2007, págs. 25-27) y LÓPEZ BETANCOURT y FONSECA LUJÁN (2016, págs. 25-28).

8 Las observaciones hechas en esta sección están inspiradas (principalmente, si bien no exclusivamente) en la reconstrucción de la legislación relevante dentro del ordenamiento jurídico mexicano proporcionada por LÓPEZ BETANCOURT y FONSECA LUJÁN (2016).
} 
individuales, los derechos humanos y, de manera relevante, la dignidad e integridad de las mujeres. [...].

$[\ldots]$

VIII. Acceder plenamente a la jurisdicción del Estado. Para garantizar ese derecho, en todos los juicios y procedimientos en que sean parte, individual o colectivamente, se deberán tomar en cuenta sus costumbres y especificidades culturales respetando los preceptos de esta Constitución. Los indígenas tienen en todo tiempo el derecho a ser asistidos por intérpretes y defensores que tengan conocimiento de su lengua y cultura (cursivas mías).

El Código nacional de procedimientos penales recoge estos derechos. En particular, el artículo 45 sobre el idioma de los actos procesales establece que “en el caso de los miembros de pueblos o comunidades indígenas, se les nombrará intérprete que tenga conocimiento de su lengua y cultura, aun cuando hablen el español, si así lo solicitan”. Además, el artículo 113, fracción XII, establece que el imputado tiene derecho "a ser asistido gratuitamente por un traductor o intérprete en el caso de que no comprenda o hable el idioma español; cuando el imputado perteneciere a un pueblo o comunidad indígena, el Defensor deberá tener conocimiento de su lengua y cultura y, en caso de que no fuere posible, deberá actuar asistido de un intérprete de la cultura y lengua de que se trate”. Finalmente, el artículo 410 sobre los “Criterios para la individualización de la sanción penal o medida de seguridad” establece que “cuando el sentenciado pertenezca a un grupo étnico o pueblo indígena se tomarán en cuenta [...] sus usos y costumbres”.

Además, el artículo 420 en materia de procedimientos especiales establece que:

Cuando se trate de delitos que afecten bienes jurídicos propios de un pueblo o comunidad indígena o bienes personales de alguno de sus miembros, y tanto el imputado como la víctima, o en su caso sus familiares, acepten el modo en el que la comunidad, conforme a sus propios sistemas normativos en la regulación y solución de sus conflictos internos proponga resolver el conflicto, se declarará la extinción de la acción penal, salvo en los casos en que la solución no considere la perspectiva de género, afecte la dignidad de las personas, el interés superior de los niños y las niñas o del derecho a una vida libre de violencia hacia la mujer.

Ahora bien, en el papel, el ordenamiento jurídico mexicano parece garantizar un reconocimiento amplio, y articulado en diferentes formas, del derecho a la diversidad cultural de los pueblos indígenas, incluso en el ámbito penal. De hecho, el conjunto de estas normas perece sugerir que en el ordenamiento jurídico mexicano se encuentran ambas las soluciones mencionadas anteriormente (§ 1). Por un lado, el artículo 420 del Código nacional de procedimientos penales proporciona la posibilidad de una forma de pluralismo jurídico e institucional, si bien bajo precisas condiciones: es decir, que el objeto del delito sean bienes jurídicos de un pueblo indígena o de uno de sus miembros, que tanto el imputado como la víctima acepten el modo en el que los sistemas normativos de la comunidad solucionan el conflicto, y que la solución considere adecuadamente la

\footnotetext{
${ }^{9}$ Los mismos derechos se les reconocen a la víctima u ofendido (artículos 109, fracción XI, y 110 sobre la designación de asesor jurídico).
} 
perspectiva de género y no afecte la dignidad de las personas, el interés superior de los niños y las niñas o el derecho a una vida libre de violencia hacia la mujer.

Por otro lado, el ordenamiento jurídico mexicano también parece admitir la posibilidad de la defensa cultural. En este sentido, en su análisis sobre "el derecho penal frente a la interculturalidad en México”, LÓPEZ BETANCOURT y FONSECA LUJÁN (2016, pág. 30) afirman que:

\begin{abstract}
Dentro del catálogo de derechos reconocidos a los indígenas, el derecho de pleno acceso a la justicia del Estado se ha concretado en tres grupos de prerrogativas con reconocimiento dispar en la legislación federal y local: a) derechos complementarios del debido proceso derivados de la diferencia de lengua y cultura (intérprete, defensor conocedor de la lengua y costumbres); $b$ ) beneficios derivados de la situación de los indígenas como grupo vulnerable (trato favorable en la sentencia y durante su ejecución, revisión oficiosa del respeto a derechos); y c) derechos derivados de la diferencia jurídico-cultural (consideración de sus especificidades culturales, costumbres o sistemas normativos).
\end{abstract}

Según estos autores, en particular, este último grupo de derechos (c) constituye el reconocimiento legislativo de un derecho a la defensa cultural e "implica asignar a los juzgadores el deber de averiguar y evaluar de oficio la posible existencia de [un] conflicto normativo”. En apoyo de su tesis, los autores recuerdan que

\begin{abstract}
la Primera Sala de la Suprema Corte de Justicia de la Nación ha señalado que los jueces penales, cuando deban determinar si una persona indígena merece ser castigada por haber incurrido en una conducta típica y punible consignada en la legislación penal aplicable, deberán "indagar cuáles son las costumbres y especificidades de la comunidad a la que [el imputado] se vincula que han podido influir en el desarrollo de los hechos enjuiciados”. Es decir, "deberá[n] tomar en cuenta [...] tanto las diferentes normas de fuente estatal aplicables como las específicas que puedan existir en la comunidad cultural del procesado con relevancia en el caso".
\end{abstract}

Al respecto, también subrayan que, ya que "parece difícil que el juzgador realice por sí mismo la indagación de las normas consuetudinarias de una comunidad indígena de la cual probablemente ni siquiera conoce el idioma”, entonces “se prevé la prueba del peritaje antropológico o cultural como la vía idónea para identificar la influencia de la cultura y las normas consuetudinarias en el caso concreto” (LÓPEZ BETANCOURT y FONSECA LUJÁN, 2016, pág. 31).

Sin embargo, López Betancourt y Fonseca Luján abordan al menos dos críticas importantes sobre la forma en que la ley mexicana toma en cuenta las diferencias culturales de los miembros de los pueblos indígenas. En primer lugar, explican que no hay claridad sobre los efectos de la defensa cultural, ya que "aunque las legislaciones afirman que los jueces y tribunales deben tomar en cuenta la especificidad cultural o las costumbres al dictar sentencia y fijar las sanciones, nada dicen sobre la ubicación en la sistemática del delito de estos elementos” (LÓPEZ BETANCOURT y FONSECA 
LUJÁN, 2016, pág. 32) ${ }^{10}$. En segundo lugar, los autores lamentan que, en la aplicación práctica de la defensa cultural, la diferencia de los indígenas "se considera primariamente una causa de vulnerabilidad”, ya que, hasta ahora, se ha intentado acomodar esta diferencia "en las categorías de la teoría del delito como causa de imputabilidad, causa de inculpabilidad por error de prohibición, y circunstancia atenuante o eximente por falta de necesidad preventiva de la pena” (LÓPEZ BETANCOURT y FONSECA LUJÁN, 2016, pág. 39). En cambio, ellos proponen enmarcar la defensa cultural en el derecho constitucional de los pueblos indígenas a sus propios sistemas normativos, de modo que esta defensa pueda ser calificada como causa de justificación debida al legítimo ejercicio de un derecho, excluyendo de raíz la antijuridicidad de la conducta (LÓPEZ BETANCOURT, FONSECA LUJÁN, 2016, pág. 34)¹1.

\title{
3 LOS DESAFÍOS “TÉCNICOS” DEL RECONOCIMIENTO DE LA IDENTIDAD CULTURAL EN EL ÁMBITO PENAL
}

A pesar del reconocimiento del derecho a la diversidad cultural a nivel internacional y nacional, la concreta garantía de este derecho en el ámbito penal tiene que lidiar con algunos obstáculos. Por un lado, como se dirá en un rato, hay diferentes opiniones sobre la deseabilidad de reconocer la diversidad cultural en el ámbito penal, cada una de las cuales tiene sus razones para discutir (§ 4). Por otro lado, hay efectivamente algunos desafíos “técnicos” - bajo el perfil jurídico, institucional e, incluso, socio-antropológico - en la realización de este reconocimiento.

En primer lugar, hay dificultades en la realización de un régimen de pluralismo jurídico e institucional. De hecho, como subrayan también LÓPEZ BETANCOURT y FONSECA LUJÁN (2016, pág. 38),

\begin{abstract}
El ejercicio [del] derecho a impartir justicia según la normativa interna de los pueblos, requiere del cumplimiento de los correlativos deberes estatales de reconocimiento de las autoridades indígenas encargadas de la función jurisdiccional autónoma, respeto a los procedimientos acordes con la normativa indígena y a las resoluciones tomadas según estos, y realización de las adecuaciones institucionales necesarias para una precisa distribución de competencias entre los órganos jurisdiccionales estatales y los indígenas.
\end{abstract}

Para este fin, recuerdan los autores, la última parte del artículo 2 constitucional, apartado A, fracción II, también señala que “La ley establecerá los casos y procedimientos de validación por los jueces o tribunales correspondientes”.

\footnotetext{
${ }^{10}$ En particular, afirman, la ley no dice "si la existencia de norma consuetudinaria justificante debe considerarse en la antijuridicidad; si la diferencia cultural cuenta para la identificación de la imputabilidad; si debe considerarse en la valoración de la culpabilidad o en qué grado puede motivar una atenuación de la pena”.

${ }^{11}$ De la opinión diametralmente opuesta es, en cambio, CARNEVALI (2007, págs. 26-28).
} 
Sin embargo, aun dejando de lado el problema de la posible falta de voluntad política para cumplir con este mandato constitucional, la idea de instituir formas de gobernanza compartida entre el Estado y los pueblos indígenas representa, en efecto, un difícil desafío de ingeniería institucional al que, hasta ahora, incluso las propuestas más serias y articuladas de "jurisdicciones multiculturales" (SHACHAR 2001) no parecen haber ofrecido respuestas decisivas. En particular, persisten problemas que no son fáciles de resolver en cuestiones como, por ejemplo, la identificación de criterios unívocos para establecer cuando un individuo pertenece un pueblo indígena y de los términos para fijar la división de responsabilidades entre el Estado y los pueblos indígenas. Además, los derechos colectivos como tales requieren que se aborden algunas cuestiones técnico-jurídicas sobre su estructura y la forma en que se ejercen. En particular, no es inequívoco cómo pueden y deben identificarse los que tienen derecho a actuar en nombre del grupo. Además, no es fácil establecer sobre la base de qué autoridad lo hacen y con qué representatividad. Finalmente, es controvertido a través de qué mecanismos se les puede y/o se les debe imputar la responsabilidad (no solo frente al Estado o terceros sino también, y sobre todo, hacia los miembros del grupo) de las acciones llevadas a cabo y de las decisiones tomadas en calidad de líderes del grupo mismo.

Similarmente, incluso la defensa cultural no está exenta de problemas, ya que la definición de los presupuestos de su aplicación depende de cómo se construyen la premisa fáctica y la premisa normativa de la decisión judicial.

En particular, bajo el perfil de los hechos, la evaluación de cualquier factor cultural potencialmente relevante para la decisión judicial puede resultar extremadamente problemática. De hecho, esta evaluación presupone que el juez verifique la existencia (desde un punto de vista objetivo) y la incidencia (desde un punto de vista subjetivo) de una pluralidad de condiciones esencialmente controvertidas. En particular, desde un punto de vista objetivo, el juez debe preguntarse, en primer lugar, si la cultura constituye efectivamente una clave para la lectura correcta del caso en el que debe pronunciarse $^{12}$. La noción de cultura, de hecho, es controvertida; por lo tanto, no es infrecuente que comportamientos culturalmente connotados no sean considerados como tales, o viceversa, que la calificación cultural se niegue a comportamientos que, en cambio, encuentran explicación (y a veces justificación) en la cultura del imputado. En segundo lugar, es necesario considerar que no está del todo claro cómo es posible establecer qué prácticas identifican rasgos característicos e inalienables de una cultura (o quién tiene derecho de establecerlo). En particular, no está del todo claro qué relevancia debe atribuirse, por ejemplo, a las prácticas controvertidas o de abandono dentro del grupo

\footnotetext{
12 Esta es la primera pregunta del “test cultural” proporcionado por RUGGIU (2012, pág. 293) para ayudar al juez en la evaluación de que relevancia reconocer a la cultura del imputado frente a un presunto delito culturalmente motivado.
} 
al que pertenece el imputado, o cuáles, entre las posibles variantes de una práctica determinada, deben ser considerados “auténticas” u “oficiales”13: de hecho, las culturas no son homogéneas ni coherentes. Además, desde el punto de vista subjetivo, el juez debe cuestionar sobre qué influencia han ejercido efectivamente los factores culturales sobre la conducta del imputado. En particular, es importante determinar si el imputado ha considerado obligatorias ciertas reglas de conducta y en qué medida. Y también, puede ser importante preguntarse si consideró las alternativas disponibles como realmente válidas, tomando en cuenta las posibles consecuencias negativas a las que le hubieran expuesto la transgresión de las reglas de conducta consideradas vinculantes dentro de su grupo cultural.

Similarmente, ni siquiera la identificación del derecho aplicable es tarea fácil, ya que las sociedades multiculturales se caracterizan a menudo por una gran pluralidad y heterogeneidad de grupos, que se refleja en la gran variedad de sistemas jurídicos que los tribunales estatales deberían tener en cuenta (PAROLARI, 2016, págs. 215, 220-231; 2018, págs. 36-37). Por lo tanto, en estos contextos, el clásico principio iura novit curia puede perder gran parte de su sentido. De hecho, parece difícil imaginar que los jueces estatales puedan aplicar las normas penales de los grupos culturales, aunque solo sea porque, verosímilmente, no las conocen adecuadamente.

Finalmente, cabe recordar que la reconstrucción de los hechos objeto de juicio (quaestio facti) y la individuación del derecho aplicable (quaestio juris) son operaciones constitutivamente interdependientes entre sí, y no son un acto de cognición sino primariamente de decisión, profundamente condicionado por presupuestos de carácter cultural (PAROLARI, 2016, págs. 206207). En particular, bajo el primer perfil, hay que tener en cuenta que la quaestio facti y la quaestio juris son el éxito de un proceso circular de condicionamiento mutuo ${ }^{14}$ : por un lado, la interpretación prima facie de los hechos sujetos a juicio, según lo informado por las partes, dirige al juez en la identificación del tipo abstracto en el que subsumir el concreto; por otro lado, al mismo tiempo, el “preconocimiento” que tiene el juez de las normas potencialmente aplicables lo guía en la identificación de los elementos fácticos relevantes, entre aquellos referidos por las partes. De hecho, es el derecho que establece si un hecho es jurídicamente relevante y qué clasificación jurídica se le debe atribuir ${ }^{15}$, y siempre es el derecho, en particular las reglas relativas a la adquisición de pruebas,

\footnotetext{
13 Poner atención a esta cuestión es verdaderamente importante ya que, como denuncia POLLIT (1997, pág. 26), raramente se cuestiona si la cultura del imputado presenta efectivamente los caracteres descritos en el procedimiento.

${ }^{14}$ MAZZARESE (1996, pág. 85) habla a este respecto de una “doble enlace” (doppio legame) entre quaestio facti y quaestio juris. Sobre las principales cuestiones problemáticas relacionadas con las nociones de quaestio facti y quaestio juris y las relaciones entre la formulación de uno y otro, véase MAZZARESE (1996, págs. 57-103).

${ }^{15}$ Como señala MAZZARESE (1996, págs. 85, 88-92), la noción de hecho resulta ser particularmente problemática sobre todo, si bien no exclusivamente, en el caso de "hechos determinados de manera evaluativa" (piénsese, por ejemplo, en cláusulas generales y términos evaluativos como diligencia o daño injusto), de "hechos psíquicos o actitudes psicológicas”
} 
a establecer cómo pueden y/o deben determinarse los hechos que son objeto de juicio (Véanse, por ejemplo, TARUFFO, 1992; FERRER BELTRÁN, 2004; MAZZARESE, 1996, págs. 92-99). Además, bajo el segundo perfil, hay que considerar las influencias culturales (más o menos explícitas) que condicionan al juez en la formulación de la premisa fáctica y de la premisa normativa en las que basa su decisión. De hecho, el juez está inevitablemente condicionado por su cultura (jurídica) cuando identifica e interpreta el derecho aplicable y cuando, a través de la lente de este derecho, reconstruye los hechos, los interpreta y establece su clasificación jurídica. En el contexto de una sociedad multicultural, sin embargo, los presupuestos culturales del juez no necesariamente coinciden con los del imputado, tanto en relación con el valor atribuido a los hechos cuanto en relación con las normas jurídicas que se asumen aplicables. Por lo tanto, para conseguir una correcta calificación jurídica de los hechos de juicio, el tribunal debe tener en cuenta el valor que el imputado atribuyó a sus acciones o comportamientos, en relación no sólo a su cultura, sino también a las normas jurídicas a las que él creía (y cree) tener que conformarse ${ }^{16}$.

En este contexto, surge la necesidad de recurrir a expertos (RENTELN, 2010, págs. 97-102), especialmente a formas de peritaje antropológico, para proporcionarle al juez asesoramiento, sea para entender correctamente los hechos, sea para conocer el derecho aplicable (si bien, al final, le compete al juez valorar la prueba de este peritaje). Además, en el proporcionar su asesoramiento, los expertos tienen que tener en cuenta los efectos de las mutuas contaminaciones interculturales que normalmente caracterizan las sociedades multiculturales, ya que el contacto intercultural afecta a las transformaciones tanto de las identidades culturales (que no son monolíticas ni estáticas) como de las normas jurídicas (sea de las estatales, sea de las de los grupos culturales). De hecho, en los contextos multiculturales, los distintos sistemas jurídicos normalmente no quedan completamente separados, sino que interactúan - si bien a menudo informalmente - entre sí, lo que termina por generar formas de derecho híbrido y en continua transformación (PAROLARI, 2016, pág. 230; 2018, págs. 36-37). Por lo tanto, es esencial que los expertos no adopten una concepción estática y de museo de las diferentes identidades culturales y los diferentes sistemas normativos/jurídicos, sino que se enfrenten a su naturaleza dinámica y cambiante, proporcionando una valoración “caso por caso” de los aspectos concretamente relevantes.

(piénsese, por ejemplo, en la determinación de malicia, de culpa o de buena fe) y de "hechos institucionales” (piénsese, por ejemplo, en la validez de un contrato, un testamento o un matrimonio).

${ }^{16}$ Cabe subrayar que, como señala PASTORE (2012, pág. 46), la “jurisdicción sensible a la cultura” debe, de hecho, estar "dirigida no solo a la cultura de las minorías, sino a todas las culturas en juego (también a la cultura de la mayoría u 'oficial'), en la perspectiva de una estrategia de ‘encuentro bidireccional’” (cursivas en el texto, traducción mía). 


\section{RAZONES EN FAVOR Y EN CONTRA DEL RECONOCIMIENTO DE LA IDENTIDAD CULTURAL EN EL ÁMBITO PENAL}

A los mencionados problemas “técnicos”, hay que añadir los posibles prejuicios ideológicos de los jueces, que, por varias razones, podrían no estar dispuestos a tomar en serio la exigencia del reconocimiento de las diversidades culturales en el ámbito penal. De hecho, se han propuesto muchos argumentos en contra de este reconocimiento ${ }^{17}$.

En primer lugar, se afirma que el reconocimiento de la identidad cultural del imputado conlleva el riesgo de un particularismo exasperado y de formas de "monoculturalismo plural” (SEN, 2006, pág. 158) en las que grupos culturales diferentes se limitan a coexistir en el aislamiento, cuando no es que entran, incluso, en conflicto. A menudo, el miedo que este riesgo pueda volverse en realidad alimenta una alternancia de instancias asimilacionistas y formas de intolerancia a los límites del racismo institucional. Sin embargo, es dudoso si la cohesión social se pone en mayor riesgo por el reconocimiento de diferencias o, por el contrario, por la falta de este reconocimiento. En muchos casos, de hecho, la obstinación en la reivindicación de sus especificidades y derechos culturales por parte de los grupos culturales y de los pueblos indígenas es directamente proporcional a la intensidad de las presiones que se ejercen sobre ellos para ser asimilados a la cultura dominante. Además, hay quien afirma que el reconocimiento de la identidad cultural del imputado puede ser considerado una expresión del pluralismo liberal en el ámbito penal. En este sentido, algunos juzgan sinónimo de intolerancia imponer a individuos de culturas diversas la adhesión a los modelos culturales hechos propios por el ordenamiento jurídico estatal, cuanto menos cuando las reglas comunes no sean “estrictamente necesarias para una cohabitación pacífica” (FERRETTI, 2004, pág. 28): cualquiera debería ser libre de vivir conforme a su propia cultura, y este derecho debería ser garantizado también en el ámbito penal, en tanto que no sean violados los derechos fundamentales de otros sujetos ${ }^{18}$.

En segundo lugar, el reconocimiento de la identidad cultural del imputado ha sido criticado porque la previsión de un tratamiento diferenciado para los imputados de culturas diversas constituye una forma de discriminación, tanto entre los imputados, como entre las víctimas. En particular, en

\footnotetext{
${ }^{17}$ Además de los argumentos que se van a mencionar en esta sección, los mayores desacuerdos se concentran, desde la perspectiva iuspenalista, en torno a las consecuencias que la defensa cultural puede producir, respectivamente, sobre los efectos preventivos, sobre la adecuación retributiva y sobre la capacidad reeducativa de la pena. Y también, es objeto de debate si la defensa cultural viola dos principios fundamentales del derecho penal: el principio de legalidad y el principio según el cual ignorantia legis non excusat.

${ }^{18}$ Escribe a propósito DE FRANCESCO (2006, pág. 138): “De frente a la multiplicación de las intervenciones, en los campos tanto jurídico como sociológico, a favor del reconocimiento de la necesidad de asegurar a las diversas culturas (así como a los denominados derechos de las minorías) un espacio de legitimización conforme a la inspiración pluralista de los sistemas contemporáneos, la idea de exceptuar la política criminal de la explicación de un rol compatible con la persecución de objetivos similares no puede no parecer intolerablemente regresiva y anacrónica” (traducción mía).
} 
relación a la condición del imputado, el hecho de que la defensa cultural pueda ser invocada no por cualquier imputado, sino sólo por quien pertenece a una cultura diversa de aquella que informa el derecho penal estatal, comportaría que para un mismo tipo penal fuesen previstas consecuencias jurídicas diferentes, dependiendo de quien cometa el delito (Sobre este punto véanse, entre otros, COLEMAN, 1996, págs. 90, 94; MONTICELLI, 2003; PHILLIPS, 2003, pág. 513; CARNEVALI, 2007). Y también, en relación a la condición de la persona ofendida, garantizar el reconocimiento de las especificidades culturales del imputado instauraría, de hecho, un régimen de menor garantía de los derechos de las víctimas de delitos culturalmente motivados respecto a los derechos de las víctimas de otros delitos: de este modo, se dice, se debilitaría la protección ofrecida por el derecho a los sujetos vulnerables (mujeres y niños/as sobre todo) de aquellos mismos grupos culturales y pueblos indígenas que se querría tutelar ${ }^{19}$. Sin embargo, el reconocimiento de la identidad cultural también podría ser considerado, por el contrario, como un instrumento para garantizar el igual tratamiento de personas de cultura diversa. De hecho, hay quien denuncia que la afirmada neutralidad del Estado de frente a las diferencias culturales es en realidad una neutralidad aparente, porque el ordenamiento jurídico del Estado expresa lo valores de la cultura dominante ${ }^{20}$. Por esta razón, se sostiene que pretender la adhesión incondicionada a las normas penales estatales por parte de individuos pertenecientes a culturas diversas significa discriminarlas respecto de aquellos que no están constreñidos a elegir entre la ley del Estado y los valores de su propia cultura ${ }^{21}$.

En tercer lugar, quien enfatiza el valor de la autonomía individual denuncia el oportunismo implícito en el uso de la cultura como pretexto para justificar conductas delictuosas (HONIG, 1997). Además, se lamenta que este uso de los argumentos culturales hace propia y contribuye a difundir una imagen estereotipada y errada de las culturas otras ${ }^{22}$. En efecto, esta segunda preocupación remite a una crítica del esencialismo cultural que merece atenta consideración (PAROLARI, 2016, págs. 4170). Sin embargo, el hecho de tener en cuenta la influencia del contexto cultural de origen sobre el

\footnotetext{
${ }^{19}$ La cuestión de los derechos de las víctimas es recurrente en la literatura sobre la defensa cultural. COLEMAN (1996) hace notar que en todos los casos en los que han sido aceptados los argumentos vertidos por la defensa cultural, la víctima pertenecía a la misma cultura del imputado. En el campo de una más amplia reflexión sobre la relación entre multiculturalismo y derechos de las mujeres, véanse también OKIN (1997); BENHABIB (2002, págs. 122-127).

${ }^{20}$ Sobre la relación entre derecho penal y cultura véase, en particular, CASTILLO ARA (2014, págs. 245-246).

${ }^{21}$ De este argumento, que caracteriza la literatura de orientación comunitarista, rinde cuenta, entre otros, BERNARDI (2006, pág. 97).

${ }^{22}$ Escribe SIKORA (2001, pág. 1708): "Muchos opositores [de la defensa cultural] creen que una defensa cultural promueve estereotipos negativos de las minoría e inmigrantes porque esta asume que la cultura de un área en particular es fácilmente identificable y que toda la gente de aquella área actuará de forma similar” (traducción mía). Y también, POLLIT (1997, pág. 27), subraya que "la argumentación basada en los derechos culturales funciona mejor en el caso de culturas que [conocemos] menos: culturas que, en nuestra ignorancia, prefiguramos como estables, fuera del tiempo, antiguas, privadas de conflictos internos, premodernas” (traducción mía).
} 
carácter y voluntad del imputado puede representar, en cambio, un medio para reconocer la manera en que, en efecto, las normas sociales, morales y jurídicas del grupo pueden condicionar las elecciones individuales de sus miembros, aunque solo sea porque la marginación en contra de quien viola tales normas puede ser para el individuo una sanción más grave que la misma sanción penal.

Finalmente, en cuarto lugar, se lamenta que la garantía del derecho a la diversidad cultural en el ámbito penal puede determinar una protección inferior de los derechos fundamentales. En particular, una preocupación recurrente es que la protección de los derechos fundamentales por el derecho y las instituciones estatales se debilite cada vez más en cuanto más aumente el espacio reconocido a otros cuerpos normativos, especialmente si el reconocimiento de este espacio pasa por formas de la autonomía normativa e institucional de los grupos culturales y pueblos indígenas. De hecho, el establecimiento de formas similares de autonomía puede dificultar la identificación e implementación de métodos efectivos de control externo sobre la garantía de los derechos fundamentales dentro de estos grupos y pueblos. En particular, se subraya la dificultad de identificar y proporcionar medios efectivos para garantizar la protección de las personas vulnerables a través de la garantía de un “derecho de salida” - es decir, el derecho de los miembros del grupo de abandonarlo cuando lo deseen - que no sea meramente formal. De hecho, hay que tener en cuenta que pertenecer a un grupo cultural o a un pueblo indígena no puede considerarse voluntario de la misma manera que la afiliación a una asociación o a un partido político: allí, uno nace y crece, condicionado por un tejido social y familiar de afectos y relaciones de poder. Por esta razón, el mero reconocimiento formal de un derecho de salida no parece representar una alternativa real para los sujetos que se encuentran en una condición de sujeción psicológica y/o de falta de independencia económica.

Ahora bien, la preocupación por la protección de los derechos fundamentales es absolutamente compartible. Sin embargo, derivar de la imprescindibilidad de esta protección una defensa sin apelación del "monismo estatal” (RUGGIU, 2017, pág. 122) - o, para decirlo de otra forma, del “centralismo jurídico” (GRIFFITHS, 1986, pág. 4) - parece no solo una obsesión anacrónica sino también una opción ideológica y política no obligada. Una obsesión anacrónica porque, a partir del periodo de la segunda posguerra, la identificación, implementación y protección de los derechos fundamentales ya no son una prerrogativa exclusiva de los Estados, sino que se encuentran determinadas por la intersección entre la esfera jurídica nacional y la internacional. Una opción ideológico-política no obligada porque, en sí mismo, nada impide que al menos podamos tratar de concebir y dibujar un pluralismo jurídico (e institucional) basado en el respeto y en la protección de los derechos fundamentales, tanto por parte de los Estados y de las instituciones internacionales, como por parte de los grupos culturales y pueblos indígenas. 
Además, cabe recordar que los conflictos entre derechos fundamentales no son algo que ocurra solamente cuando sea implicado el derecho a la diversidad cultural, ya que siempre derechos (de personas) diferentes pueden entrar en conflicto en los casos concretos. Sin embargo, hay un mecanismo para resolver estos conflictos: la ponderación. De hecho, la ponderación es la vía elegida también por el derecho internacional. En este sentido, la Declaración universal sobre la diversidad cultural (UNESCO, 2001) establece, por un lado, que "la defensa de la diversidad cultural es un imperativo ético, inseparable del respeto de la dignidad de la persona humana” (artículo 4$)^{23}$ y que “los derechos culturales son parte integrante de los derechos humanos” (artículo 5); y por otro lado, al mismo tiempo, la misma Declaración precisa que "nadie puede invocar la diversidad cultural para vulnerar los derechos humanos garantizados por el derecho internacional, ni para limitar su alcance” (artículo 4). Similarmente, en la Declaración sobre los derechos de los pueblos indígenas (ONU, 2007), al reconocimiento de cada derecho colectivo siempre sigue la aclaración que el ejercicio de este derecho en los casos concretos no debe perjudicar los derechos humanos individuales reconocidos por el derecho internacional. Además, como se ha visto (§ 2), la misma Constitución mexicana reconoce a los pueblos indígena el derecho a "aplicar sus propios sistemas normativos en la regulación y solución de sus conflictos internos, sujetándose a los principios generales de esta Constitución, respetando las garantías individuales [y] los derechos humanos” (artículo 2, apartado A, fracción II).

Claramente, en la ponderación del derecho a la diversidad con otros derechos fundamentales, hay que considerar cual es el bien jurídico tutelado, si hay daños a terceros, cual es el grado de asimilación del imputado y si la acción representa un delito también en el grupo de origen. Sin embargo, la valoración de estos elementos tiene que ser realizada con una sensibilidad intercultural, para evitar superponer los valores del grupo dominante a aquellos de otros grupos culturales.

\section{CONCLUSIONES}

La cuestión del respeto por las diferencias culturales en ámbito penal es probablemente una de las cuestiones más controvertidas en el debate sobre la tutela del derecho a la diversidad cultural. En particular, todavía no existe consenso unánime sobre la viabilidad y la deseabilidad de establecer

\footnotetext{
${ }^{23}$ En este artículo también se afirma que la defensa de la diversidad cultural "supone el compromiso de respetar los derechos humanos y las libertades fundamentales, en particular los derechos de las personas que pertenecen a minorías y los de los pueblos autóctonos”.
} 
formas de pluralismo jurídico o de autorizar el recurso a la defensa cultural en los tribunales estatales. No obstante, hay Estados que, como México, ya han tomado, al menos en parte, este camino.

Ciertamente, tanto el pluralismo jurídico como la defensa cultural plantean “desafíos técnicos” que deben tomarse en serio. Con referencia al pluralismo jurídico, piénsese, por ejemplo, a los desafíos de ingeniería institucional relacionados con la realización de formas de gobernanza compartida entre el Estado y los grupos culturales o pueblos indígenas, así como a la necesidad de abordar de manera efectiva los problemas relacionados con la estructura y a la forma de ejercer los derechos colectivos. Por otro lado, con referencia a la defensa cultural, piénsese, por ejemplo, a la dificultad de integrar la evaluación de los factores culturales potencialmente relevantes dentro del proceso (circular) de construcción de la premisa fáctica y de la premisa normativa de la decisión judicial. Dificultades que incluyen, entre otras cosas, la exigencia que al juez posea - directamente o por el medio de formas especializadas de peritaje - las necesarias competencias antropológicas y un conocimiento adecuado del derecho del grupo o del pueblo indígena al que el imputado pertenece (siempre teniendo en cuenta, además, las continuas transformaciones de las identidades culturales y de los sistemas normativos generados por el contacto intercultural).

Además, se deben proporcionar respuestas convincentes a las críticas normativas, sin duda razonables, que se han dirigidas al pluralismo cultural en el ámbito penal, con referencia, en particular, al mantenimiento de la cohesión social, al riesgo de esencialismo cultural, al respeto del principio de igualdad y a la protección de los derechos fundamentales (incluso, y sobre todo, de los derechos de los miembros más vulnerables de los mismo grupos culturales y pueblos indígenas).

Sin embargo, ni los “desafíos técnicos” ni las criticas normativas parecen completamente invencibles. De hecho, aunque la identificación de posibles “equilibrios avanzados” (CAMMARATA, 2017, pág. 112) entre el pluralismo jurídico y la protección de los derechos fundamentales es ciertamente un objetivo difícil de alcanzar, no es, sin embargo, algo a priori imposible. En particular, el mecanismo para resolver los potenciales conflictos entre el derecho a la diversidad cultural y otros derechos fundamentales ya está identificado por el mismo derecho constitucional e internacional, y consiste en la ponderación.

La verdadera cuestión no es, entonces, si tenemos que garantizar el derecho a la diversidad cultural, incluso en el ámbito penal, ya que a esto nos obliga el derecho internacional y constitucional, sino, más bien, cómo identificar los términos para formar un régimen jurídico respetuoso, al mismo tiempo, de la especificidad de las diferentes culturas jurídicas y de la igualdad de todos los seres humanos en los derechos fundamentales. 


\section{REFERENCIAS}

BASILE, F. (2008) Immigrazione e reati “culturalmente motivati”. Il diritto penale nelle società multiculturali europee. Milano: CUEM.

BENHABIB, S. (2002) The Claims of Culture: Equality and Diversity in the Global Era. Princeton: Princeton University Press; trad. it., La rivendicazione dell'identità culturale. Eguaglianza e diversità nell'era globale. Bologna: il Mulino, 2005.

BERNARDI, A. (2006) Modelli penali e società multiculturale. Torino: Giappichelli.

BERNARDI, A. (2007) 'L'ondivaga rilevanza penale del 'fattore culturale', Politica del diritto, 38(1), págs. 3-48.

BREMS, E. (2017) 'Legal Pluralism as a Human Right and/or as a Human Rights Violation', en CORRADI, G., BREMS, E. y GOODALE M. (Coords.), Human Rights Encounter Legal Pluralism. Normative and Empirical Approaches. Oxford and Portland: Hart Publishing, págs. 23-39.

CAMMARATA, R. (2010) 'La Dichiarazione delle Nazioni Unite sui diritti dei popoli indigeni alla luce della cosmovisione Maya. Un successo e una sfida per i diritti umani’, Thule - Rivista italiana di studi americanistici, 26/29, págs. 91-113.

CAMMARATA, R. (2012) Indegno a chi? Diritti e discriminazioni allo specchio. Torino: Giappichelli.

CAMMARATA, R. (2017) 'Differenze culturali e diritti fondamentali: le sfide del diritto, il ruolo della politica’, Notizie di Politeia, 128, págs. 111-114.

CARNEVALI, R. (2007) 'El multiculturalismo: un desafío para el Derecho penal moderno', Política Criminal, 3, A6, págs. 1-28.

CASTILLO ARA, A. (2014) 'La ponderación de las valoraciones culturales en el error de prohibición’, Revista de Derecho (Valdivia), 27(2), págs. 243-267.

COLEMAN, D. L. (1996) 'Individualizing Justice through Multiculturalism: The Liberals' Dilemma’, Columbia Law Review, 96(5), págs. 1093-1167.

DE FRANCESCO, G. (2006) 'Multiculturalismo e diritto penale nazionale', en BERNARDI A. (Coord.), Multiculturalismo, diritti umani, pena. Milano: Giuffrè, págs. 137-163.

DE MAGLIE, C. (2010) I reati culturalmente motivati. Ideologie e modelli penali. Pisa: Edizioni ETS.

FERRAJOLI, L. (2007) Principia iuris. Teoria del diritto e della democrazia. Roma-Bari: Laterza.

FERRAJOLI, L. (2018) Manifesto per l'uguaglianza. Roma-Bari: Laterza.

FERRER BELTRÁN, J. (2004) Prova e verità nel diritto. Bologna: il Mulino. 
FERRETTI, M. P. (2004) 'Tre modi di intendere le differenze culturali', en RICCIARDI M. y DEL BO, C. (Coords.), Pluralismo e libertà fondamentali. Milano: Giuffrè, págs. 5-30.

FISCHER, M. (1998) 'The Human Rights implications of a 'Cultural Defense’', Southern Carolina Interdisciplinary Law Journal, 6, págs. 663-702;

FOBLET, M.-C. y RENTELN, A. D. (Coords.) (2009) Multicultural jurisprudence. Comparative Perspectives on the Cultural Defense. Oxford and Portland: Hart Publishing.

FOBLETS, M.-C. (1998) 'Les délits culturels: de la répercussion des conflits de culture sur la conduite délinquante. Réflexions su l'apport de l'anthropologie du droit à un débat contemporain', Droit et Culture, 35, págs. 195-222.

GIANFORMAGGIO, L. (2005) Eguaglianza, donne e diritto. Bologna: il Mulino.

GOLDING, M. P. (2002) ‘The Cultural Defense’, Ratio Juris, 15(2), págs. 146-158.

GRIFFITHS, J. (1986) 'What is Legal Pluralism?’, The Journal of Legal Pluralism and Unofficial Law, 24, págs. 1-55.

HONIG, B. (1997) 'Complicating Culture', Boston Review, 22 [online]. Disponible en: http://bit.ly/36hrc3H (accedido: 10 de junio 2019).

HURTADO POZO, J. (2001) ‘El indígena ante el Derecho Penal: caso peruano', en MORENO HERNÁNDEZ M. (Coord.), La ciencia penal en el umbral del siglo XXI, México, págs. 29-45. Disponible en: http://bit.ly/38ou0hy (accedido: 20 de mayo 2019).

LILLO, R. (2015) 'Justicia penal y derechos indígenas. El largo camino del reconocimiento de la diversidad’, Revista Austral de Ciencias Sociales, 28, págs. 99-115.

LÓPEZ BETANCOURT, E. y FONSECA LUJÁN, R.C. (2016) 'El derecho penal frente a la interculturalidad en México’, Revista de investigación en Derecho, Criminológica y Consultoría Jurídica, 9(18), págs. 19-40.

LYMAN, J. C. (1986) ‘Cultural Defense: Viable Doctrine or Wishful Thinking?’, Criminal Justice Journal, 9(1), págs. 87-117.

MAUREIRA PACHECO, M. (2005) 'Política criminal e indigenismo. Fundamentos jurídicoteórico de la política criminal indigenista’, Indiana, 22, págs. 189-213.

MAZZARESE, T. (1996) Forme di razionalità delle decisioni giudiziali. Torino: Giappichelli.

MAZZARESE, T. (2014) 'El derecho a ser diversos en las sociedades multiculturales', en DOUGLAS PRICE, J. y DUQUELSKY, D. (Coords.), Ponencias XXVII Jornadas Argentinas de Filosofía Jurídica y Social. Multiculturalismo, interculturalidad y derecho. Buenos Aires: Editorial Ministerio de Justicia y Derechos Humanos de la Nación, págs. 3-18.

MAZZARESE, T. (2018) 'I migranti e il diritto ad essere diversi nelle società multiculturali delle democrazie costituzionali’, en CERRINA FERONI, G. y FEDERICO, V. (Coords.), Strumenti, 
percorsi e strategie dell'integrazione nelle società multiculturali. Napoli: Edizioni Scientifiche Italiane, págs. 63-85.

MONTICELLI, L. (2003) 'Le ‘cultural defenses' (esimenti culturali) e i reati ‘culturalmente orientati’. Possibili divergenze tra pluralismo culturale e sistema penale’, Indice penale, 6(2), págs. 535-585.

OKIN, S. M. (1997) 'Is Multiculturalism Bad for Women?’, Boston Review, n. 22 [online]. Disponible en: http://bit.ly/38fvSsK. (accedido: 10 de junio 2019).

OLIVER, M. (1988) 'Immigrant Crimes: Cultural Defense - A Legal Tactic’, L. A. Times, 15 de Julio, págs. 1, 30.

PAROLARI, P. (2011) 'Delitos culturalmente motivados. Un nuevo desafío del multiculturalismo a los derechos fundamentales’, en NARVÁEZ HERNÁNDEZ J. R. (Coord.), Interpretación jurisprudencial. Memorias del II Simposio Internacional de Jurisprudencia. D.F. (México): Suprema Corte de Justicia de la Nación, págs. 236-281.

PAROLARI, P. (2016) Culture, diritto, diritti. Diversità culturale e diritti fondamentali negli stati costituzionali di diritto. Torino: Giappichelli.

PAROLARI, P. (2018) 'Migrazioni, interlegalità, pluralismo giuridico’, Rivista di Filosofia del diritto, 7(1), págs. 33-50.

PASTORE, Baldassare [2012], Impegni ermeneutici: il diritto e le tensioni del pluralismo culturale, en “Ars Interpretandi”, 1 (2012), págs. 41-58.

PÉREZ DE LA FUENTE, O. (2012) 'Delitos culturalmente motivados. Diversidad cultural, derecho e inmigración’, European Journal of Legal Studies, 5(1), págs. 60-94.

PHILLIPS, A. (2003) 'When Culture Means Gender: Issue of Cultural Defence in the English Courts’, The Modern law Review, 66, págs. 510-531.

POLLITT, K. (1997) 'Whose Culture?', Boston Review, n. 22 [online]. Disponible en: http://bit.ly/36jCLaC (accedido: 10 de junio 2019).

RENTELN, A. D. (2004) The Cultural Defense. Oxford: Oxford University Press.

RENTELN, A. D. (2010) The Cultural Defense: Challenging the Monocultural Paradigm, en FOBLETS, M.-C., GAUDREAULT-DESBIENS, J.-F. y RENTELN, A. D. (Coords.), Cultural Diversity and the Law. State Responses from Around the World. Bruxelles: Bruylant, págs. 791817.

RUGGIU, I. (2012) Il giudice antropologo. Costituzione e tecniche di composizione dei conflitti multiculturali. Milano: Franco Angeli.

RUGGIU, I. (2017) 'La cultura tra teoria dei diritti fondamentali e teoria del pluralismo giuridico', Notizie di Politeia, 128, págs. 119-124. 
SANZ MULAS, N. (2018) Los delitos culturalmente motivados, Valencia (ES): Editorial Tirant lo Blanch.

SEN, A. (2006) Identity and Violence. The Illusion of Destiny. New York-London: W.W. Norton \&Company.

SHACHAR, A. (2001) Multicultural Jurisdictions. Cultural Differences and Women's Rights. Cambridge: Cambridge University Press.

SHEYBANI, M.-M. (1987) 'Cultural Defense: One Person's Culture Is Another's Crime’, Loyola Los Angeles International and Comparative Law Journal, 9(3), págs. 751-783.

SIKORA, D. W. (2001) 'Differing Cultures, Differing Culpabilities? A Sensible Alternative: Using Cultural Circumstances as a Mitigating Factor in Sentencing', Ohio State Law Journal, 62(5), págs. 1695-1728.

SOTOMAYOR ACOSTA, J. O. (1996) 'La responsabilidad penal del indígena en Colombia. Entre el mundo real y el mundo posible’, Jueces para la democracia, 26, págs. 89-96.

TARUFFO, M. (1992) La prova dei fatti giuridici. Milano: Giuffrè.

UPRIMNY, R. (2011) 'The Recent Transformation of Constitutional Law in Latin America: Trends and Challenges’, Texas Law Review, 89, págs. 1587-1609.

VALENZUELA REYES, M. (2003) 'Derechos de los pueblos indígenas en el contexto internacional, especialmente en lo relativo a los aspectos penales’, Revista de Estudios Criminológicos y Penitenciarios, 6, págs. 9-36.

VAN BROECK, J. (2001) 'Cultural Defence and Culturally Motivated Crimes (Cultural Offences)', European Journal of Crime, Criminal Law and Criminal Justice, 9(1), págs. 1-32.

VOLPP, L. (1994) '(Mis)identifying culture: Asian Women and the 'Cultural Defense’', Harvard Women’s Law Journal, 17, págs. 57-101.

YOUNG, C. (1992) ‘Equal Cultures - or Equality?’, Washington Post, 29 de Marzo, pág. C5. 